CASE REPORT

\title{
Spurious rise in the automated platelet count because of bacteria
}

\section{N Kakkar}

J Clin Pathol 2004;57:1096-1097. doi: 10.1136/icp.2004.016758

The era of automation in haematology, although improving the accuracy and precision of results, has also introduced the laboratory haematologist to a vast array of spurious parameters. The identification of these results is important so that inappropriate management decisions are avoided. The case presented here illustrates a spuriously raised automated platelet count resulting from bacterial overgrowth in the blood sample.

$\mathrm{T}$ he use of automated analysers in haematology laboratories is now the rule rather than the exception. These instruments have enhanced the precision of results and, with optimal quality control measures in the laboratory, have improved the accuracy of tests. However, there are a variety of conditions where automated parameters may be fictitious. Such situations demand careful attention, because vital management decisions may be taken based on these erroneous results. ${ }^{1}$ Both the laboratory operators of these instruments and clinicians must be aware of the possibility of spurious data from automated laboratories. A case of an artefactual rise in the automated platelet count is presented here.

"The use of automated analysers in haematology laboratories is now the rule rather than the exception"

\section{CASE REPORT}

A 39 year old woman was admitted to the coronary care unit of our hospital with sudden onset of breathlessness. On examination, she was tachypnoeic and restless. Respiratory examination revealed bilateral crepitations. Prosthetic valve sounds could be well heard. She had tachycardia but her heart rate was regular. A diagnosis of acute pulmonary oedema was made and supportive treatment was initiated. She was being followed up after mitral valve replacement, the surgery having been performed 11 years previously for chronic rheumatic valvular heart disease. The presentation with pulmonary oedema was attributed to her failure to increase the dosage of the prescribed diuretic (Furosemide) from 40 to $80 \mathrm{mg}$ each day. As part of her initial investigation, a complete blood count was requested. The complete blood count reported by the Advia-60 (Bayer, Baroda, India), the three part differential automated analyser in use in our laboratory, revealed a haemoglobin of $128 \times 10^{9} \mathrm{~g} / \mathrm{litre}$; a total leucocyte count of $8.5 \times 10^{9}$ /itre, with a normal differential leucocyte count; mean corpuscular volume of $78 \mathrm{fl}$; mean corpuscular haemoglobin of $26.1 \mathrm{pg}$, and a mean corpuscular haemoglobin concentration of $33.6 \%$. The platelet count was $1152 \times 10^{9}$ /litre. However, a review of the peripheral blood smear revealed a platelet count of approximately $130-150 \times 10^{9}$ /itre, a discrepancy of nearly
$1000 \times 10^{9}$ /itre compared with the automated value. Numerous rod shaped bacteria were present in clumps and were also seen lying singly (fig 1). A few intraneutrophilic bacteria were also present. The white blood cells showed pronounced artefactual changes, with nuclear distortion and cytoplasmic vacuolation. The bacteria seen in the peripheral smear were thought to be responsible for the spuriously raised platelet count. Further clinical details on the patient were sought, in view of the history of prosthetic valve surgery and thereby a high risk of bacteraemia. She had no symptoms or signs of infective endocarditis and the valve function, both clinically and on echocardiography, was satisfactory. No other obvious focus of infection could be identified.

It was subsequently discovered that the sample was drawn early in the morning in the emergency room. There was then a delay of eight hours before it reached the laboratory because of a wrongly filled requisition form. The blood sample was kept at room temperature until this time.

A repeat sample was taken, which revealed an automated platelet count of $158 \times 10^{9}$ /itre. The peripheral blood smear made from the repeat sample revealed no bacteria.

It was concluded that bacteria seen in the blood smear of the patient's initial sample were the result of bacterial overgrowth in the blood sample caused by a delay in analysis rather than bacteraemia in the patient. Overgrowth was probably aided by the ambient temperature of approximately $35^{\circ} \mathrm{C}$ prevalent at the time of sampling.

The patient was discharged in a satisfactory condition after five days in the hospital. She was asymptomatic at the first outpatient follow up.

\section{DISCUSSION}

Data from modern automated haematology analysers, if backed up by good quality control measures, are generally considered reliable, although spurious results may be generated at times. Among these, falsely high platelet counts may have important clinical consequences, possibly also masking severe underlying thrombocytopenia. Thus, all unexpectedly high platelet counts must be verified by examination of the blood smear by an experienced morphologist. $^{2}$ Spuriously raised platelet counts can be seen in conditions with pronounced microcytosis (haemoglobin $\mathrm{H}$ disease), microangiopathic haemolytic anaemia, burns, leukaemia, and lymphoma. ${ }^{1}$ The presence of bacteria in the peripheral blood smear has been reported on many occasions in the literature.

Various bacteria have been seen in blood smear examinations, ${ }^{3}{ }^{4}$ and at times the peripheral blood smear has been the first clue to the presence of infection in patients. ${ }^{5-7}$ However, there are fewer reports on spuriously raised automated platelet counts as a result of bacteraemia. Gloster et al reported two patients with positive blood cultures for Escherichia coli and Klebsiella pneumoniae who also had bacteria seen in their peripheral blood smears. Their platelet counts 


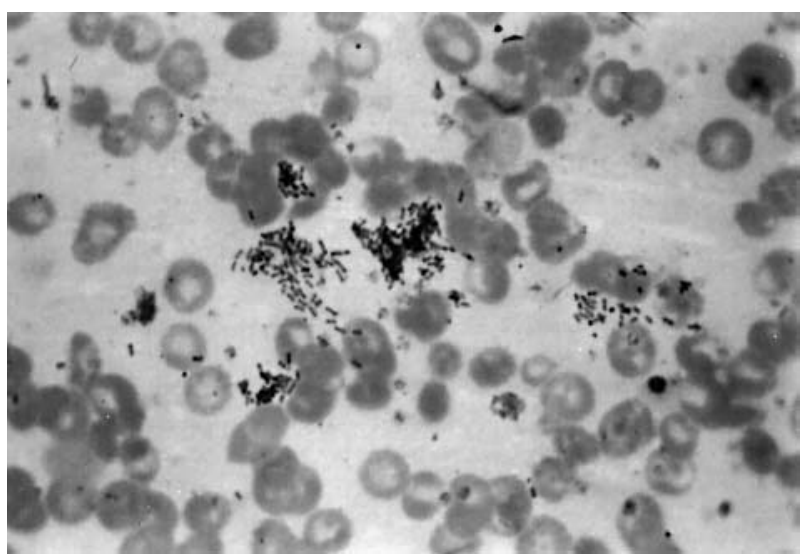

Figure 1 Bacteria seen in the peripheral blood smear (Leishman's stain; original magnification, $\times 1000)$.

generated by the Ortho-ELT-8 machine were spuriously raised. ${ }^{8}$

When present, true bacteraemia usually results in a small number of bacteria in the blood smear, with an intraneutrophilic location, unlike the case presented here which had numerous clumps of bacteria. This case draws attention to the importance of pre-analytical variables with respect to laboratory testing. In the case of an anticipated delay in the processing of blood samples, refrigeration at $4{ }^{\circ} \mathrm{C}$ greatly improves the stability of test results, a recommendation that was not followed in this case.

"Samples with unexpected blood counts in relation to the clinical setting or those that generate specific flags necessitate a careful review of the blood smear"

This case also highlights the contribution that the now often ignored blood smear can make to detecting abnormalities that the much heralded chip may miss at times. High output laboratories with modern automated haematology analysers have been devising methods to lessen the need to review blood smears. This seems a natural consequence of the advanced technology that these analysers use. However, it must be remembered that samples with unexpected blood counts in relation to the clinical setting or those that generate specific flags necessitate a careful review of the blood smear. Ignoring this could lead to missing out on vital, clinically useful information.

Standard operating procedures for automated analysers must include definite guidelines on situations in which a
Take home messages

- This report describes a spuriously raised automated platelet count resulting from bacterial overgrowth in the blood sample

- This case draws attention to the importance of preanalytical variables with respect to laboratory testing overgrowth occurred because there was a delay in testing and the sample was kept at $35^{\circ} \mathrm{C}$ instead of being refrigerated

- All unexpectedly high platelet counts must be verified by examination of the blood smear by an experienced morphologist

- Standard operating procedures for automated analysers must include definite guidelines on situations in which a blood smear review is mandatory

blood smear review is mandatory. This will aid in reducing spurious results.

To conclude, pre-analytical variables need careful attention when investigating aberrant results that the laboratory haematologist encounters.

Correspondence to: Dr N Kakkar, Department of Pathology, Christian Medical College and Hospital, Ludhiana-141 008, Punjab, India; n_kakkar@satyam.net.in

Accepted for publication 20 April 2004

\section{REFERENCES}

1 Bain BJ. Detection of erroneous blood counts. In: Blood cells: a practical guide, 3rd ed. London: Blackwell Science, 2002:155-74.

2 Perkins SL. Blood and bone marrow examination. In: Greer JP, Foerester J, Rodgers GM, et al. Wintrobe's clinical hematology, 10th ed. Baltimore: Williams and Wilkins, 1999:9-35.

3 Posner MR, Berk SL, Rice PA. Pneumococcal bacteremia diagnosed by peripheral blood smear in multiple myeloma. Arch Intern Med 1978;138:1720-1.

4 Pederson G, Schonheyder HC, Nielsen LC. Capnocytophaga canimorsus bacteremia demonstrated by a positive blood smear. A case report. APMIS 1993; 101:572-4

5 Fife A, Hill D, Barton C, et al. Gram negative septicaemia diagnosed on peripheral blood smear appearances. J Clin Pathol 1994;47:82-4.

6 Alvarez Elcoro S, Sifuentes Osorio J. Clostridium perfringens bacteremia in prosthetic valve endocarditis. Diagnosis by peripheral blood smear. Arch Intern Med 1984; 144:849-50.

7 Supparatpinyo K, Sirisanthana T. Disseminated Penicillium marneffei infection diagnosed on examination of a peripheral blood smear of a patient with human immunodeficiency virus infection. Clin Infect Dis 1994; 18:246-7.

8 Gloster ES, Strauss RA, Jimenez JF, et al. Spurious elevated platelet counts associated with bacteremia. Am J Hematol 1985;18:329-32. 